\title{
Implantación de un contrato conductual en estudiantes universitarios: una experiencia en la asignatura 'Estructura y función del cuerpo humano'
}

\author{
A. Aracil-Marco ${ }^{a}$, J. Gallar-Martínez ${ }^{\text {b }}$
}

Introducción. Se describen los resultados académicos de los estudiantes de la asignatura 'Estructura y función del cuerpo humano' impartida en Ciencias del Deporte tras firmar un contrato conductual, y se comparan con los del curso académico previo. Sujetos y métodos. Se estableció un contrato conductual por el que cada estudiante podía obtener beneficios académicos, ligados a la consecución de objetivos a lo largo del curso. Se analizó el efecto de esta intervención sobre los resultados académicos, así como sobre la satisfacción de los estudiantes con la asignatura. Resultados. El contrato conductual fue elegido por el $83 \%$ de los estudiantes. De ellos, sólo un 13\% no alcanzó los objetivos mínimos. La tasa de éxito (estudiantes aprobados respecto a los presentados) aumentó del 42 al 68\%, respecto al curso previo, obteniéndose además una mejora en las calificaciones. Más del 95\% de los estudiantes que superaron la asignatura en el curso 2006-2007 había participado en el contrato, y de los participantes en el mismo el 74\% superó la asignatura en el curso académico. Asimismo, el índice de calidad percibida por los estudiantes ascendió significativamente en casi un punto respecto al curso anterior. Conclusiones. Los contratos conductuales parecen herramientas pedagógicas útiles para mejorar el rendimiento académico y la calidad percibida de la docencia universitaria.

Palabras clave. Aprendizaje activo. Competencias transversales. Contrato conductual. Contrato de contingencias. Gestión del aprendizaje. Rendimiento académico.

\section{Implementation of a contingency contract in undergraduate students: an experience on the subject 'Structure and function of the human body'}

Introduction. Academic results of undergraduate students of the subject 'Structure and function of the human body' in a Sports Sciences degree, are compared before and after the implementation of a contingency contract. Subjects and methods. A contingency contract was implemented during the academic year 2006-2007. Voluntary participants in the experience were able to obtain various benefits depending on their achievement of academic objectives. Results of this intervention on academic achievement were compared among participants and non-participants, as well as with the previous academic year. Results. $83 \%$ of the students participated voluntarily in the experience. Only 13\% of them did not reach the minimum objectives to obtain any benefit. Taking together, academic results improved in the year of the intervention compared with the previous year. Over $74 \%$ of the participants in the experience passed the subject during the academic year 2006-2007. Similarly, subjective quality perception on the teaching of the subject significantly improved after the implementation of the contingency contract. Conclusions. Contingency contracts seem to be useful instruments to improve academic achievement of undergraduate students.

Key words. Psychological contract, contingency contract, active learning, generic competences, learning management, academic achievement

\section{Introducción}

La importancia sociosanitaria actual que, desde un punto de vista esencialmente preventivo, puede tener la realización habitual de actividad física, así como los riesgos inherentes a la misma, justifica que se deba exigir de los futuros profesionales de las Ciencias de la Actividad Física y del Deporte un conocimiento suficiente de diferentes aspectos biomédicos. Esta necesidad formativa
Área de Educación Física. Departamento de Psicología de la Salud.

b Departamento de Fisiología. Universidad Miguel Hernández. Elche, Alicante, España.

Correspondencia Dr. Adolfo Aracil Marco. Área de Educación Física y Deportiva. Departamento de Psicología de la Salud. Universidad Miguel Hernández. Campus de Elche, edif. Torrevaillo. Avda. Universidad, s/n. E-03202 Elche (Alicante).

E-mail

fito@umh.es

Agradecimientos A E. Cervelló, F.J. Moreno, F.J. Vera-Martínez, J.L. López-Elvira, R. Costa y E León, de la UMH, por sugerencias, y a M.C. Acosta (UMH), J.A. Moreno (Universidad de Murcia) y M. González (H.G.U. de Alicante), por la lectura crítica del manuscrito.

El proyecto piloto objeto de este trabajo ha sido galardonado con el Premio a la Innovación Docente, dentro de la $3 .^{\text {a }}$ convocatoria de Premios del Consejo Social de la UMH de Elche, gracias al cual se financió en parte su realización. Algunos resultados preliminares se expusieron en el XVII Congreso de la SEDEM

(Tenerife, Octubre 2007). 
Tabla I. Descripción de la procedencia académica (modo de acceso y rama, en su caso) de los estudiantes matriculados en CAFD en cada curso académico.

\begin{tabular}{lcc}
\hline & Curso 2005-2006 & Curso 2006-2007 \\
\hline Prueba de acceso a la universidad & 84 & 81 \\
\hline Arte & 2 & 11 \\
\hline Humanidades & 10 & 26 \\
\hline Ciencias Sociales & 21 & 14 \\
\hline Científico-tecnológica & 15 & 30 \\
\hline Ciencias de la Salud & 36 & 7 \\
\hline Formación Profesional & 9 & 0 \\
\hline Con título universitario & 2 & 4 \\
\hline Mayores 25 años & 3 & 8 \\
\hline Estudios extranjeros / otros & 2 & \\
\hline \begin{tabular}{l} 
CAFD: Ciencias de la Actividad Física y del Deporte. Todos los datos se expresan en porcentajes, calculados sobre el total de estudiantes \\
\hline
\end{tabular} & & \\
\hline
\end{tabular}

choca frontalmente con los posibles conocimientos previos con los que los estudiantes acceden al primer curso de la licenciatura en Ciencias de la Actividad Física y del Deporte (CAFD). Así, por ejemplo, menos de la mitad de los estudiantes de nuevo ingreso en primer curso accede a la titulación desde las opciones científico-tecnológicas (14-15\%) o de salud (30-36\%) del Bachillerato, mientras que algo más del $50 \%$ accede desde la opciones de ciencias sociales, humanidades, pruebas para mayores de 25 años o Formación Profesional (Técnico Superior en Animación de Actividades Físicas y Deportivas) (Tabla I), en cuyas enseñanzas mínimas no se contemplan determinadas materias básicas (biología, física, matemáticas, etc.) que, si bien no son prerrequisito legal, sí constituyen una base fundamental para cursar adecuadamente algunas materias de los primeros cursos de CAFD (biología y fisiología celular, anatomía funcional, fisiología humana, biomecánica, etc.). Esta formación dispar de los estudiantes que acceden a la titulación puede explicar por qué en algunas asignaturas la tasa de rendimiento académico, entendida como el porcentaje de estudiantes matriculados que superan una asignatura en el curso académico, pueda ser, en ocasiones, anormalmente baja.

Para intentar mejorar los resultados académicos en la asignatura 'Estructura y función del cuerpo humano', una asignatura troncal de primer curso de la licenciatura en CAFD en nuestra universidad, y con el fin de iniciar su adaptación a las nuevas metodologías docentes exigidas por el Espacio Europeo de Educación Superior (EEES), durante el pasado curso 2006-2007 se implantó, como experiencia piloto, la firma voluntaria por parte del estudiante de un contrato conductual. En el presente artículo se describe dicha experiencia y se comparan los resultados académicos obtenidos por los estudiantes con los del curso inmediatamente precedente, en el que sólo se usó una metodología expositiva. 


\section{Sujetos y métodos}

Se ofreció a los estudiantes matriculados durante el curso 2006-2007 en la asignatura 'Estructura y función del cuerpo humano', de la titulación Licenciado en CAFD, la posibilidad de participar voluntariamente en esta experiencia piloto, mediante la cual se podían obtener beneficios académicos diferentes, ligados a la realización de tareas y consecución de objetivos a lo largo del período lectivo del curso académico. Tanto las tareas como los beneficios fueron explícitos desde el comienzo de curso, y la voluntad del estudiante de participar en esta experiencia se plasmó en un contrato conductual (que denominamos el 'contrato de aprendizaje'), que había de ser firmado expresamente.

Además del resto de las actividades académicas previstas (incluida la evaluación), los estudiantes tuvieron que participar en:

- Un seminario sobre técnicas de estudio.

- Un seminario sobre planificación de su estudio.

- Un seminario/debate sobre reflexiones de la importancia de una buena formación para su futuro éxito profesional, tomando como ejemplo el análisis de comentarios sobre profesionales de éxito.

- Una serie de tutorías mensuales de repaso de conocimientos y evaluación, realizadas en grupo. Para ello, los participantes se distribuyeron en cinco grupos, compuestos de 30-35 estudiantes.

Cada grupo realizaba una tutoría, de unas 3 horas de duración, una vez al mes. Antes de cada sesión, los estudiantes debían:

- Realizar esquemas o resúmenes de los temas impartidos hasta ese momento en las clases regulares de la asignatura.

- Responder por escrito una serie de preguntas referidas a los objetivos del aprendizaje explicitados para cada uno de los temas impartidos.

- Razonar la respuesta a dichas preguntas.

En cuanto a los beneficios que se debían conseguir, la asistencia a cada sesión suponía para el estudiante obtener un punto. Por la realización de los esquemas y las preguntas se obtenía medio punto, y durante la sesión se conseguía medio punto adicional por responder correctamen- te, y de memoria, a una pregunta elegida aleatoriamente por el profesor de entre las entregadas por escrito por el estudiante. En total, pues, se podía obtener un máximo de dos puntos por sesión. Además, durante el primer cuatrimestre, no presentar los esquemas o las preguntas y no responder correctamente a la pregunta no se penalizó. Sin embargo, con el ánimo de estimular la preparación de los estudiantes con vista a la evaluación final, durante el segundo cuatrimestre sí se aplicaba una penalización, de manera que se restaba medio punto por cada acción no realizada. En otras palabras, asistir a la tutoría sin haber realizado los esquemas y sin saberse las preguntas equivalía a perder el punto por la asistencia, con lo que podía llegar a no obtenerse ningún punto en dicha sesión.

Durante el desarrollo de las sesiones de tutoría presencial, el estudiante tenía acceso a la evolución de su puntuación, de modo que cada participante tenía un feedback inmediato sobre la repercusión de su acción, y podía comparar su progresión con la del grupo. Al final del curso se evaluó el porcentaje de puntos obtenidos por cada estudiante sobre el total de los posibles, y en función de ello se otorgaron recompensas. La tabla II resume el sistema de obtención de puntos, y la tabla III las recompensas relacionadas con el logro de puntos.

Se calculó el porcentaje de estudiantes que aceptaron participar en el estudio, y se analizó su nivel de logro de objetivos y sus resultados académicos, que se compararon con los de quienes decidieron no participar. Se calcularon las siguientes tasas de rendimiento académico:

- Tasa de éxito, definida como la proporción de estudiantes calificados aptos sobre el total de estudiantes que se presentaron a evaluación en las convocatorias de junio y septiembre de cada curso académico.

- Tasa de rendimiento, entendida como proporción de estudiantes calificados aptos a lo largo del curso académico sobre el total de estudiantes matriculados al comienzo del curso.

- Distribución de las calificaciones obtenidas por los estudiantes que superaron la asignatura.

Además, se obtuvo el índice medio de satisfacción con la asignatura, calculado anualmente por la Oficina de Gestión de la Calidad de la Universidad Miguel Hernández (UMH) mediante encuestas de calidad percibida de la docencia por 
Tabla II. Descripción del sistema de puntuación aplicado a los firmantes del contrato de aprendizaje.

\begin{tabular}{|c|c|c|}
\hline \multicolumn{3}{|l|}{ Seminarios introductorios } \\
\hline Técnicas de estudio & Asistencia: 1 punto & \\
\hline Planificación del propio estudio & Asistencia: 1 punto & $\begin{array}{l}\text { Planificación del tiempo: } \\
\text { - Hecha: } 1 \text { punto } \\
\text { - Caso contrario: } 0 \text { puntos }\end{array}$ \\
\hline $\begin{array}{l}\text { Reflexión sobre la importancia del estudio } \\
\text { para el futuro ejercicio profesional }\end{array}$ & Asistencia: 1 punto & $\begin{array}{l}\text { Comentarios de dos textos: } \\
\text { - Hechos: } 1 \text { punto } \\
\text { - Caso contrario: } 0 \text { puntos }\end{array}$ \\
\hline Puntos posibles: & 3 & 2 \\
\hline
\end{tabular}

Subtotal (S): 5

Tutorías de repaso/evaluación

\begin{tabular}{|c|c|c|}
\hline \multirow{2}{*}{$(n=7)$} & \multirow{2}{*}{ Asistencia: 1 punto } & $\begin{array}{l}\text { Esquemas de los temas vistos y preguntas } \\
\text { de repaso: } \\
\text { - Hecho: } 0,5 \\
\text { - Caso contrario: } 0 \text { (1Q), }-0,5 \text { (2Q) }\end{array}$ \\
\hline & & $\begin{array}{l}\text { Respuesta a la pregunta al azar: } \\
\text { - Correcta: 0,5 } \\
\text { - Incorrecta/no contesta: } 0 \text { (1Q), }-0,5(2 \mathrm{Q})\end{array}$ \\
\hline \multirow[t]{2}{*}{ Puntos posibles: } & 7 & 7 \\
\hline & & Subtotal (T): 14 \\
\hline
\end{tabular}

1Q: primer cuatrimestre; $2 \mathrm{Q}$ : segundo cuatrimestre.

parte de los estudiantes. Esta evaluación se realiza mediante un cuestionario autoadministrado, al final de cada cuatrimestre. Los estudiantes la realizan anónimamente de forma presencial o vía web, tras validación. La satisfacción con la asignatura se evalúa mediante siete ítems más una opción de respuesta abierta. La valoración de una asignatura está comprendida entre 0 y 10 .

Igualmente, se compararon los datos globales de rendimiento académico del curso 2006-2007, en el que se realizó la experiencia, con los del curso inmediatamente precedente. El análisis de los datos se realizó con el software Sigmastat (SPSS, Inc.), utilizando el test estadístico adecuado en cada caso. Se consideró que las diferencias eranestadísticamente significativas cuando $p<0,05$. Los gráficos se dibujaron con Sigmaplot (SPSS, Inc.).

\section{Resultados}

Durante el curso 2006-2007, el 83\% de los 175 estudiantes matriculados en la asignatura deci- 


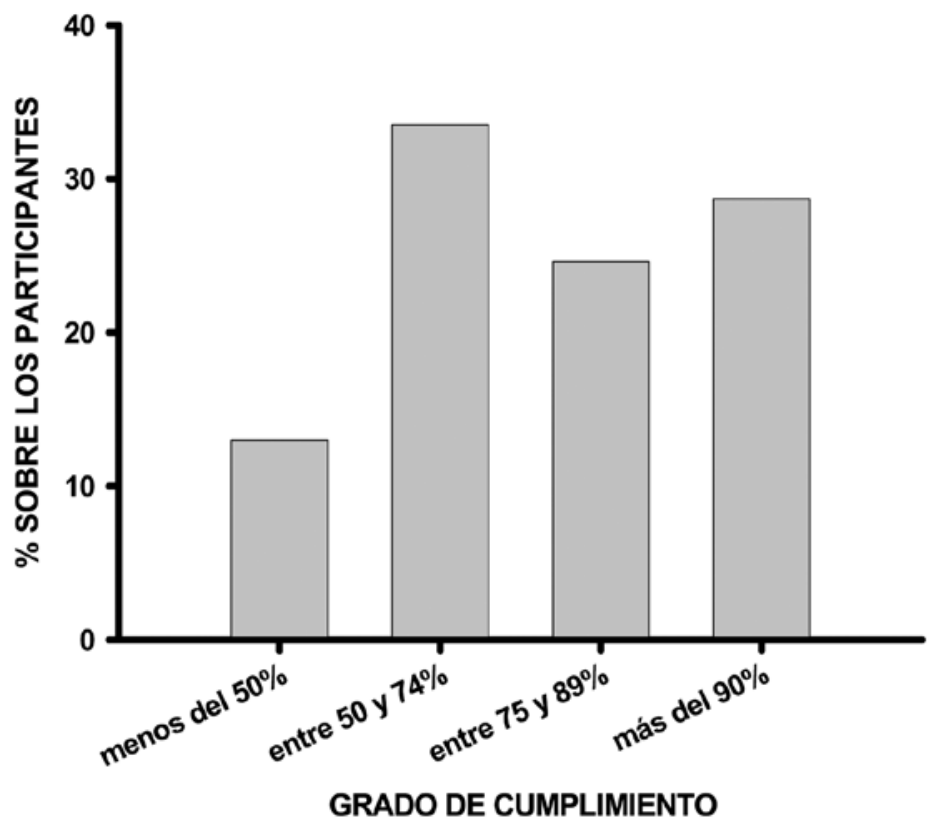

Figura 1. Grado de cumplimiento de los objetivos del 'contrato de aprendizaje'. Se ha representado el porcentaje de estudiantes participantes que lograron puntos suficientes para obtener los beneficios asignados a cada nivel de cumplimiento de objetivos previamente establecido.

Tabla III. Beneficios obtenidos en función del porcentaje de puntos logrados a lo largo del período lectivo respecto al $n$. $^{\circ}$ máximo de puntos que hay que obtener.

\begin{tabular}{ll}
\hline $50 \% \quad$ & No obtenía ningún beneficio y se \\
& consideraba que había renunciado \\
al contrato
\end{tabular}

\section{Se le guardaba la nota obtenida en los parciales aprobados hasta la convocatoria de septiembre}

$\begin{array}{ll} & \text { Se le guardaba la nota obtenida } \\ & \text { en los parciales aprobados hasta } \\ & \text { la convocatoria de septiembre y } \\ & \text { se mejoraba su calificación final } \\ & \text { en 0,5 puntos }\end{array}$

Se le guardaba la nota obtenida en los parciales aprobados hasta $\geq 90 \%$ la convocatoria de septiembre y se mejoraba su calificación final en 1 punto dió participar en esta experiencia de innovación docente. De los 146 participantes en el contrato, el $87 \%$ alcanzó porcentajes variables de puntos con los que sí se obtenían beneficios (Fig. 1), y sólo un $13 \%(n=19)$ no consiguió los puntos mínimos establecidos en el contrato de aprendizaje, por lo que, de acuerdo con las bases del éste, se consideraron como no participantes a efectos del resto de los análisis.

La tasa de éxito aumentó significativamente en el curso 2006-2007, en el que se implantó el contrato, en comparación con el curso previo (Fig. 2a). Por el contrario, la tasa de presentados disminuyó desde el 73,2\% hasta el 51,2\% $\left(p<0,001, \chi^{2}\right)$, lo que posiblemente explica que la tasa de rendimiento académico fuera un $56 \%$ en el curso 2006-2007, que ascendió sólo cinco puntos respecto al $51 \%$ obtenido en el curso previo (Fig. 2a). Si bien esta diferencia no fue estadísticamente significativa $(p=0,435)$, sobrepasar la tasa de rendimiento el 55\% (valor estándar para este indicador en el sistema de financiación por objetivos de nuestra comunidad autónoma) supuso una mejora cualitativamente relevante. La distribución de las calificaciones entre los es- 


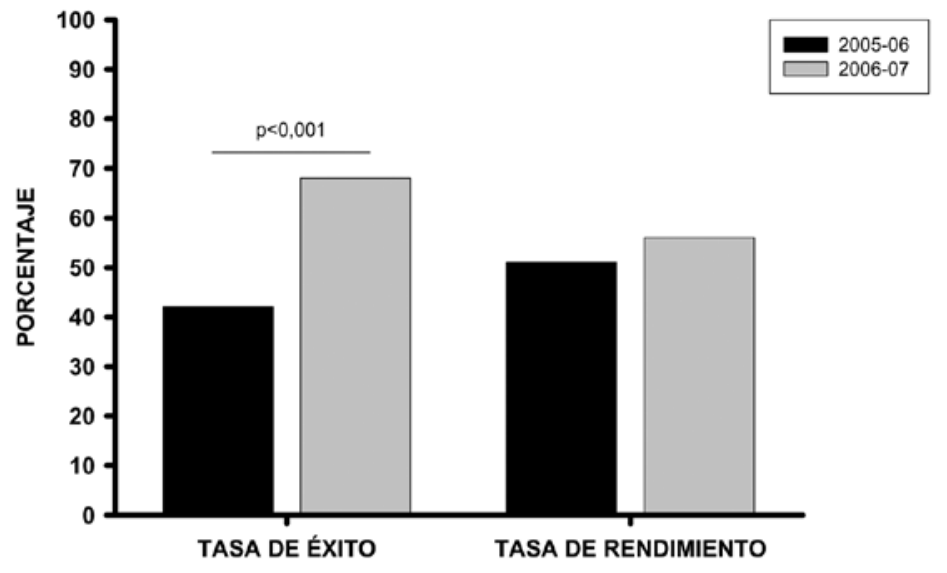

b

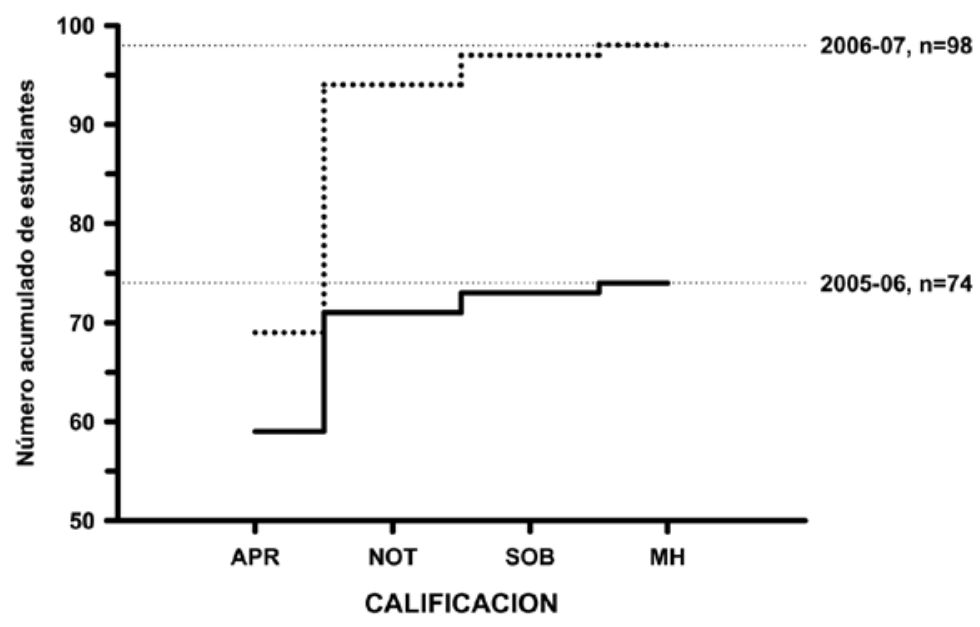

Figura 2. Resultados académicos de los estudiantes antes y después de implantar el contrato conductual. a) Tasas de éxito y de rendimiento en los cursos 2005-06 y 2006-07; b) Distribución de las calificaciones entre los estudiantes que superaron la asignatura en cada curso académico. Se muestra el número acumulado de estudiantes que superaron la asignatura con una determinada calificación, durante los cursos académicos 2005-2006 (trazo continuo) y 2006-2007 (trazo discontinuo). APR: aprobado; NOT: notable; SOB: sobresaliente; $\mathrm{MH}$ : matrícula de honor.

tudiantes que superaron la asignatura también tendió a mejorar respecto al curso previo (Fig. $2 b)$, y se observó un aumento de estudiantes calificados con aprobado o notable.

El efecto positivo encontrado en las tasas académicas del curso académico 2006-2007, tras establecer el contrato, se debió a la mejora de los resultados académicos de los participantes en el mismo. Así, se comprobó que el 95,9\% (94 de 98) de los estudiantes que superaron la asignatura correspondía a los que habían participado en el contrato. De igual modo, la tasa de éxito de los participantes (entendida como el porcentaje de participantes en el contrato que aprobaron respecto a los que se presentaron a evaluación) fue significativamente mayor que la de quienes no participaron (87,7 frente $8,3 \%$, respectivamente, $p$ $<0,001, \chi^{2}$ ) (Fig. 3a). En el mismo sentido, la tasa de rendimiento (porcentaje de participantes aprobados sobre el total de participantes) calculada para los participantes fue significativamente más alta que la tasa de rendimiento de los estudiantes que no participaron (porcentaje de no participantes aprobados sobre el total de no participantes), obteniéndose valores de 48,4 y $3,3 \%$, respectivamente $\left(p<0,001, \chi^{2}\right)$ (Fig. 3a). 


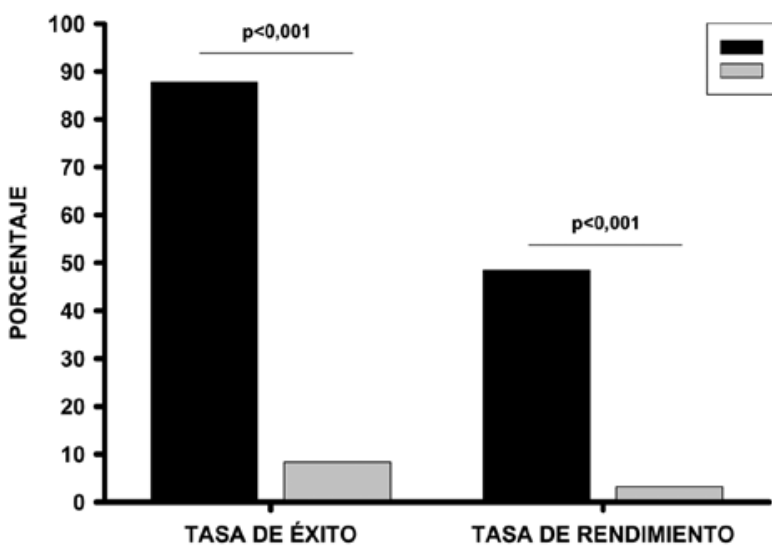

b

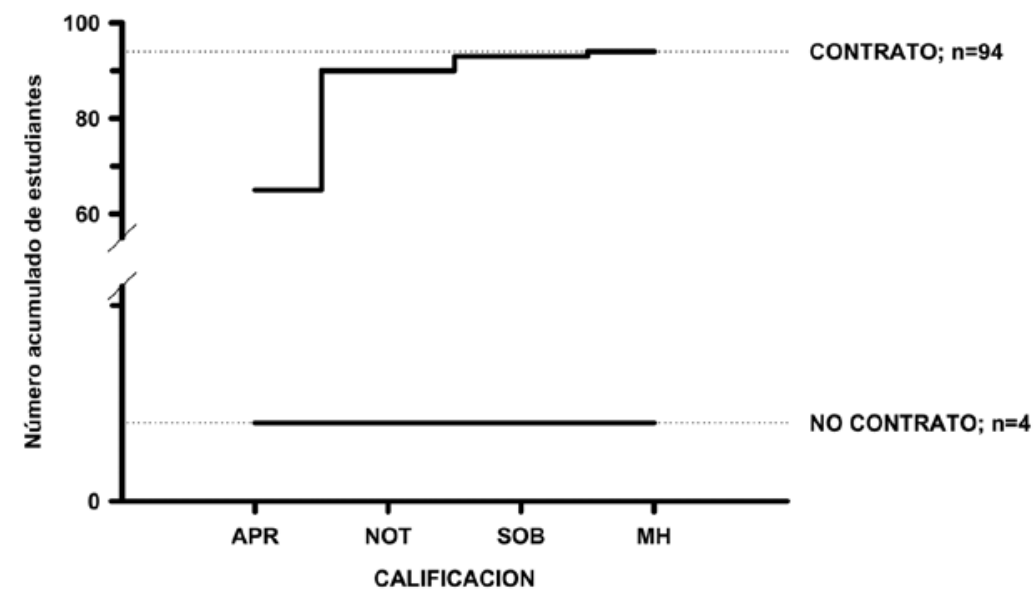

Figura 3. Efecto de la participación en el contrato conductual sobre el rendimiento académico durante el curso 2006-2007. Se comparan los valores obtenidos por los estudiantes que sí participaron en el contrato $(n=146)$ con los de aquellos que no participaron $(n=19)$. a) Tasas de éxito y de rendimiento de los participantes y no participantes en el contrato de aprendizaje. Ambas fueron significativamente superiores en los participantes en el contrato. Los valores de $p$ fueron calculados con el test de $\chi^{2}$; b) Distribución de las calificaciones obtenidas por los participantes (trazo superior) y no participantes (trazo inferior) que superaron la asignatura. Los cuatro estudiantes que superaron la asignatura sin haber participado en el contrato obtuvieron una calificación de aprobado. APR: aprobado; NOT: notable; SOB: sobresaliente; MH: matrícula de honor.

El índice de calidad percibida de la asignatura durante el curso 2006-2007 ascendió respecto al curso previo $(8,52$ frente a 7,$69 ; p<0,05, t$ de Student), siendo ese año la asignatura de primer curso de CAFD mejor valorada por los estudiantes.

\section{Discusión}

La adaptación al EEES implica la transformación del entorno educativo universitario para poten- ciar una enseñanza más centrada en el estudiante. Sin embargo, ese tipo de enseñanza, que se ha mostrado tanto o más eficiente que la 'clásica', no es esperable que se desarrolle espontáneamente en el estudiante, por lo que es necesario recurrir a estrategias pedagógicas que estimulen la implicación del estudiante en su propia progresión académica [1], particularmente en casos como el descrito en el presente trabajo, en el que la heterogeneidad de conocimientos previos de los estudiantes es muy amplia, lo que condiciona invariablemente los resultados académicos. 
Los contratos conductuales (contratos psicológicos o de contingencias) son potentes herramientas psicológicas destinadas a promover un cambio pactado y sostenido en la conducta de un sujeto [2]. Se basan en el principio del reforzamiento positivo que se administra de modo sistemático cuando se producen determinadas conductas, previamente descritas en un documento (el 'contrato') escrito y firmado por las partes. Se utilizan para ayudar al sujeto -especialmente sujetos con baja capacidad de autorreforzamiento- a iniciar y mantener conductas concretas, establecer los criterios que debe conseguir y clarificar las consecuencias positivas que tiene comprometerse con una determinada conducta, o las repercusiones negativas que tiene el romper con lo pactado.

Además de su reconocido uso en el ámbito meramente educativo, los contratos conductuales se han aplicado con éxito para corregir conductas de riesgo en ámbitos que oscilan desde la mejora de la seguridad laboral [3] a la salud [4-6]. Incluso, la reciente reforma del sistema del programa Medicaid del estado de West Virginia (EE.UU.) [7] y los diferentes programas de atención integral al médico enfermo (PAIME) se basan en este mismo procedimiento [8].

Aunque la comparación del rendimiento académico en dos cursos diferentes puede estar sometida a diferentes sesgos no controlados en el presente trabajo (como la variación en la composición efectiva de cada grupo, el nivel real de conocimientos previos de los estudiantes, su motivación, la experiencia y motivación del propio profesor, etc.), los resultados de nuestra experiencia parecen confirmar la utilidad del uso de los contratos conductuales como herramientas favorecedoras de la adherencia del estudiante al estudio programado y activo, descrita en otros niveles educativos [9].

De nuestros resultados, en los que ha mejorado fundamentalmente la tasa de éxito en los participantes en el contrato de aprendizaje, parece deducirse que esta estrategia pedagógica permite al estudiante hacer un mejor seguimiento de su progresión académica, de modo que éste se presenta a examen cuando considera que está efectivamente preparado para superarlo, y opta por lo contrario cuando considera que su nivel de conocimientos no es aún el adecuado.

Dada su utilidad, parece oportuna la implementación de este tipo de estrategias pedagógi- cas desde los primeros cursos, con la finalidad de intentar motivar al estudiante y fomentar su adherencia al estudio desde el comienzo de su formación universitaria, así como para incrementar su reflexión sobre su propia progresión académica (cuestión que se ha planteado como un determinante de su desarrollo profesional futuro) [10] o para la adquisición de competencias transversales, como la responsabilidad y motivación en el trabajo, la capacidad de aprendizaje continuo, la capacidad de organización, etc., que se han identificado, en el caso de todas las profesiones estudiadas [11] como las más valoradas por sus futuros empleadores.

\section{Bibliografía}

1. Michael J. Where's the evidence that active learning works? Advan Physiol Educ 2006; 30: 159-67.

2. Labrador FJ, Cruzado JA, Muñoz M. Manual de técnicas de modificación y terapia de conducta. Madrid: Pirámide; 1997.

3. Walker A, Hutton DM. The application of the psychological contract to workplace safety. J Safety Res 2006; 37: 433-41.

4. García-Zurdo R, García-Jiménez MV. Deshabituación del tabaco mediante un programa basado en el cambio de criterio. Psicothema 2000; 12 (Suppl 2): 257-60.

5. Virués J. Análisis funcional y tratamiento de un paciente con graves problemas de conducta diagnosticado de trastorno límite de la personalidad. Int J Clin Health Psychol 2004; 4: 207-32.

6. Gorski JA, Slifer KJ, Towsend V, Kelly-Suttka J, Amari A. Behavioral treatment of non-compliance in adolescents with newly acquired spinal cord injuries. Pediatr Rehabil 2005; 8: 187-98.

7. Steinbrook R. Imposing personal responsibility for health. N Engl J Med 2006; 355: 753-6.

8. Colegio Oficial de Médicos de Barcelona. Programa PAIME. URL: http://paimm.fgalatea.org/docs/consentiment_cast.pdf [23.05.2008].

9. Miranda A, Jarque S, Tarrasa R. Interventions in school settings for students with ADHD. Exceptionality 2006; 14: 35-52.

10. Roma J. La reflexión como eje central del desarrollo profesional. Educ Med 2006; 9: 30-6.

11. Gómez JM, Galiana D, García M, Carrasquilla C, Romero MR. Competencias profesionales en los titulados UMH. Elche: Universidad Miguel Hernández; 2006. 